\title{
From Nash and Brown to Maynard Smith: Equilibria, Dynamics and ESS*
}

\author{
J. HOFBAUER \\ Institut für Mathematik, Universität Wien, Vienna, Austria \\ (Received: 26 February 2000, \\ Accepted in revised form: 3 July 2000)
}

\begin{abstract}
Nash's three proofs for the existence of equilibria in strategic games correspond to three dynamics: The best response dynamics (equivalent to Brown's fictitious play), the smoothed best response dynamics, and the Brown-von Neumann-Nash dynamics. We prove that an equilibrium which is evolutionarily stable as defined by Maynard Smith is (globally) asymptotically stable for each of these three dynamics.
\end{abstract}

Keywords: Evolutionarily stable strategy, asymptotic stability, game dynamics

\section{Introduction}

When Maynard Smith and Price invented the concept of an evolutionarily stable strategy in 1973, they most likely did not envisage the big success and that they would create an entire new field: evolutionary game theory. Because of its simplicity and wide applicability this purely phenotypical concept has been adopted by many theoretical biologists in studying evolutionary phenomena. A look into journals like JTB or Selection shows how much this concept has penetrated evolutionary theory. Moreover it has turned out that in more sophisticated models, an equilibrium satisfying the ESS conditions often remains stable in some sense. For example, in the standard dynamic model for haploids, the replicator dynamics, an ESS is asymptotically stable. Similar results due to Maynard Smith, Lessard, Weissing, Cressman and others show this also for genetic models for diploids. I refer here only to Hofbauer and

\footnotetext{
* This paper is dedicated to John Maynard Smith at the occasion of his 80th birthday. It follows a lecture delivered at the Math Dept in Vienna on 23 April 1997. Some of the results in this paper were obtained during a stay at the Collegium Budapest in 1994/95, following an invitation by Eörs Szathmáry. I am indebted to Jörgen Weibull for sending me a copy of Nash's thesis. I thank two anonymous referees for their useful comments.

Corresponding author: J. Hofbauer, Institut für Mathematik, Universität Wien, Strudlhofgasse 4, A-1090 Vienna, Austria
}

Sigmund (1998) for a review of the present state of art in this area.

In this paper I establish a link back to the origins of (noncooperative) game theory: In fact, the replicator dynamics written down by Taylor and Jonker in 1978 was not the first game dynamics. The first dynamic models were created around 1950 by G. W. Brown as devices for computing the equilibrium strategies in zero-sum games. In the modern view of evolutionary game theory which comprises both biological and economic ideas, these early dynamics are used as population (or learning) models of agents who behave more rationally than biological replicators or imitators.

In this paper we show that Maynard Smith's ESS notion helps also towards understanding these early dynamics for general, non-zero-sum games. We prove that an ESS is asymptotically stable also for these first dynamic models due to Brown and other economically motivated dynamics.

The paper is structured as follows: We reexamine the three proofs which Nash gave for the existence of equilibria (section 2) and explain how they relate to the game dynamics introduced by Brown, von Neumann, and Fudenberg and Levine (section 3). Section 4 contains the main results of this paper: the (global) stability of ESS. In section 5 analogs of Fisher's fundamental theorem (increase of mean payoff) are given. We conclude with more general dynamics. 


\section{Nash equilibria}

We restrict to finite symmetric 2 person games. Such a game is described by an $n \times n$ payoff matrix $A$ $=\left(a_{i j}\right)$. The real number $a_{i j}$ denotes the payoff which strategy $i$ receives when playing against $j$. We assume that there is a large population of players, each committed (for some time) to one of the pure strategies. Let $x_{i}=x_{i}(t) \geq 0$ be the frequency of strategy $i$ in the population at time $t$.

The probability simplex $\Delta \subset \mathrm{R}^{n}$ (of dimension $n-1)$ is then the set of all possible population profiles $x=\left(x_{1}, \ldots, x_{n}\right)$ (with $x_{i} \geq 0$ and $\left.\sum_{i=1}^{n} x_{i}=1\right)$ which correspond to the mixed strategies in classical game theory. ${ }^{1}$

A point $\hat{x} \in \Delta$ is a (Nash) equilibrium (point) if

$$
\hat{x} \cdot A \hat{x} \geq x \cdot A \hat{x} \quad \forall x \in \Delta
$$

or equivalently

$$
\hat{x} \cdot A \hat{x} \geq(A \hat{x})_{i} \quad \forall i .
$$

Another equivalent condition is

$$
\begin{array}{ll}
\hat{x} \cdot A \hat{x}=(A \hat{x})_{i} \forall i & \text { with } \hat{x}_{i}>0 \quad \text { and } \\
\hat{x} \cdot A \hat{x} \geq(A \hat{x})_{i} \forall i & \text { with } \hat{x}_{i}=0 .
\end{array}
$$

The existence of such points is not obvious and was proved by Nash (in the more general context of finite $N$ person games). Actually he gave three different proofs in (Nash, 1950a, b, 1951).

Theorem 2.1 (Nash 1950/1951): Every game has at least one equilibrium point.

1 Besides the conception of ESS there are two features in Maynard Smith's EGT that distinguish it from the classical game theoretic literature say up to 1990: The emphasis of symmetric games and the view of a whole population of agents playing the same game. It came as a big surprise when Leonard (1994) pointed to the last chapter in Nash's thesis (1950b) which was not included in the final publication from 1951 where such a population interpretation (mass action interpretation) is given: equilibria are simply equilibria (= stationary points) of the BR dynamics. Also symmetric games are emphasised in Nash (1950b, 1951).

2 Nash (1950a) thanks 'David Gale for suggesting the use of Kakutani's theorem to simplify the proof'. It may be interesting to note that Kakutani's motivation was to give a simpler proof of the minimax theorem, i.e. the existence of equilibria in zero-sum games.

\subsection{The first proof}

Nash's brief note in PNAS 1950 introduces the notion of noncooperative $N$-person games, the concept of equilibrium and the following existence proof. The definition (1) means that $\hat{x}$ is best reply to itself. Hence, if we denote by

$$
\begin{aligned}
B R(x) & =\underset{y \in \Delta}{\arg \max } y \cdot A x= \\
& =\left\{y \in \Delta: y \cdot A x=\max _{z \in \Delta} z \cdot A x\right\} \subseteq \Delta
\end{aligned}
$$

the set of all (mixed) best responses to $x$ (which forms a subsimplex of $\Delta$ ) then (1) is equivalent to

$$
\hat{x} \in B R(\hat{x}) .
$$

The existence of such an $\hat{x}$ follows now directly from Kakutani's (1941) fixed point theorem for setvalued maps. $^{2}$

\subsection{Second proof}

In his unpublished thesis Nash (1950b) essentially considers a family of continuous functions $b_{\varepsilon}(\varepsilon>0)$ with

1. each $b_{\varepsilon}$ is a continuous map from $\Delta$ into $\Delta$ and

2. for each $x \in \Delta$, all limit points of $b_{\varepsilon}(y)$, as $\varepsilon \rightarrow 0$ and $y \rightarrow x$, are contained in $B R(x)$.

By Brouwer's fixed point theorem each of these approximate best response maps has a fixed point $\hat{x}_{\varepsilon}$ in $\Delta: b_{\varepsilon}\left(\hat{x}_{\varepsilon}\right)=\hat{x}_{\varepsilon}$. Taking an accumulation point $\hat{x}$ of these $\hat{x}_{\varepsilon}$, as $\varepsilon \rightarrow 0, \hat{x} \in B R(\hat{x})$ follows from the second property above.

Actually Nash (1950b), see also Leonard (1994, p. 500 , footnote 18 ), considers the particular family (or rather the sequence with $\varepsilon=1 / n$ )

$$
\begin{gathered}
b_{\varepsilon}(x)_{i}=\frac{c_{i}(x)}{\sum_{k} c_{k}(x)} \text { with } \\
c_{i}(x)=\left[(A x)_{i}-\max _{k}(A x)_{k}+\varepsilon\right]_{+},
\end{gathered}
$$

where $u_{+}=\max (u, 0)$ denotes the positive part. Only strategies which lose at most $\varepsilon$ compared to the maximal payoff are used in this approximate best response. 
More interesting and even smooth versions of such perturbed best-response maps are obtained by regularizing the optimization problem (3) which defines Nash equilibria: Consider the modified payoff function

$$
F(z, x)=z \cdot A x+\varepsilon v(z)
$$

where $v$ is a strictly concave function. More precisely we require that $v$ : int $\Delta \rightarrow \mathrm{R}$ is twice differentiable, the second derivative $v^{\prime \prime}(x)$ is negative definite for each $x$ in the interior of $\Delta$ and $\left|v^{\prime}(x)\right| \rightarrow \infty$ as $x$ approaches the boundary of $\Delta$. Typical examples are

$$
v(z)=\sum_{i} \log z_{i}
$$

and

$$
v(z)=-\sum z_{i} \log z_{i} .
$$

The latter choice has the advantage that the maximizer

$$
b_{\varepsilon}(x)=\underset{z \in \Delta}{\arg \max } F(z, x)
$$

can be explicitly computed as

$$
b_{\varepsilon}(x)_{i}=\frac{\exp \left(\frac{1}{\varepsilon}(A x)_{i}\right)}{\sum_{j} \exp \left(\frac{1}{\varepsilon}(A x)_{j}\right)}
$$

In any case the assumptions on $v$ imply that the maximizer $b_{\varepsilon}(x)$ in (9) is unique, depends smoothly on $x$ and $\varepsilon$, and approaches $B R(x)$ as $\varepsilon \rightarrow 0$. This elegant modification of Nash's (1950b) ansatz arose recently from a stochastic learning model for games, see Fudenberg and Levine (1998, chs 4 and 8).

\subsection{Third proof}

Nash's final proof from the Annals of Mathematics (1951) is much more streamlined and is reproduced in many texts on game theory, e.g. Owen (1982). It

3 The BR map leads to a somewhat trivial dynamical behaviour: for generic games and generic initial conditions, an orbit either becomes fixed on a pure strategy equilibrium or cycles ultimately between finitely many pure strategies. The perturbed BR maps will behave similarly for small $\varepsilon$. On the other hand, the Nash map (11) can lead to chaotic dynamics even for 2 strategies. simply applies Brouwer's fixed point theorem to the continuous map $f: \Delta \rightarrow \Delta$ defined by

$$
f(x)_{i}=\frac{x_{i}+k_{i}(x)}{1+\sum_{j=1}^{n} k_{j}(x)}
$$

where

$$
k_{i}(x)=\left[(A x)_{i}-x \cdot A x\right]_{+}
$$

and $u_{+}=\max (u, 0)$. This map is similar to Nash's perturbed BR map (5) but now all strategies doing better than average are considered. It is easy to see that $\hat{x}$ is a fixed point of $f$ if and only if $k_{i}(\hat{x})=0$ for all $i$, i.e. $\hat{x}$ is an equilibrium of the game.

\section{Dynamics}

Summarizing, these three proofs correspond to three maps on $\Delta$ :

1. the (multivalued) BR map $x \rightarrow B R(x)$;

2. the perturbed BR maps $x \rightarrow b_{\varepsilon}(x)$;

3. the map $x \rightarrow f(x)$.

Iterating these maps leads to dynamical systems on $\Delta$ in discrete time. Now continuous time dynamics usually behave more reasonably. ${ }^{3}$ Hence we consider the differential equations corresponding to the above three maps.

1. The best response dynamics (BR)

$$
\dot{x} \in B R(x)-x ;
$$

2. the perturbed (or smoothed) BR dynamics $\left(\mathrm{BR}_{\varepsilon}\right)$

$$
\dot{x}=b_{\varepsilon}(x)-x ;
$$

3. the BNN dynamics

$$
\dot{x}_{i}=k_{i}(x)-x_{i} \sum_{j=1}^{n} k_{j}(x) \text {. }
$$

While the second of these, (14), with $b_{\varepsilon}$ defined by (9), was introduced rather recently, see Fudenberg and Levine (1998, ch. 4), the other two, (13) and (15) go back to George W. Brown, the pioneer of game dynamics. (15) appears in Brown and von 
Neumann $(1950)^{4}$ for symmetric zero-sum games for which $k_{i}(x)=\left[(A x)_{i}\right]_{+}$. They show convergence to equilibrium for these games. In this dynamics the proportions of the pure strategies that are below average decrease at the same rate. Only strategies doing better than average can increase. The right-hand side of (15) is not smooth but still Lipschitz continuous. Hence the differential equation (15) has a unique solution to each initial condition.

The best response dynamics (BR) was formulated in the above form by Gilboa and Matsui (1991): In a very large population, in each small time interval a few players revise their strategy and switch to a best reply against the current population profile $x$. These players are rational, but myopic: they do not anticipate the results of their actions. With a different interpretation as a learning process, but in a mathematically equivalent form, the best response dynamics was already introduced as fictitious play by Brown $(1949,1951)$.

Due to the discontinuity or possible multivaluedness of $B R(x),(13)$ is actually a differential inclusion. Existence of solutions (these are Lipschitz functions $x(t)$ satisfying the inclusion (13) for almost all times $t$ ) follows from the general theory. On the other hand, one can explicitly construct piecewise linear solutions (in a not necessarily unique way) through each initial condition. It is shown in Hofbauer (1995), that it is sufficient to consider these piecewise linear solutions.

One of the attractive features of the smoothed best response map (9) is that because of our assumptions on $v, b_{\varepsilon}$ is a smooth function of $x$ and hence (14) has unique solutions, in contrast to the discontinuous differential equation (BR).

\section{Evolutionarily stable strategies}

According to the definition of Maynard Smith and Price (1973) and Maynard Smith (1982), a mixed strategy $\hat{x}$ is an evolutionarily stable strategy (ESS) if

$$
\text { (i) } \quad x \cdot A \hat{x} \leq \hat{x} \cdot A \hat{x} \quad \forall x \in \Delta \text {, and }
$$

4 Nash submitted his paper (1951) in October 1950, after spending the summer at RAND, see Leonard (1994, p. 499, footnote 17). Brown worked at RAND from 1948 to 1952 . It seems not impossible that Nash's third proof was inspired by the Brown-von Neumann paper. (ii) $x \cdot A x \leq \hat{x} \cdot A x \quad$ if there is equality in (i).

The first condition (i) is simply Nash's definition (1) for an equilibrium. It is well known that $\hat{x}$ is an ESS, iff $\hat{x} \cdot A x>x \cdot A x$ holds for all $x \neq \hat{x}$ in a neighbourhood of $\hat{x}$, see Hofbauer and Sigmund (1998), Maynard Smith (1982), or Weibull (1995). For an interior equilibrium $\hat{x}$, the equilibrium condition $\hat{x} \cdot A \hat{x}$ $=x \cdot A \hat{x}$ for all $x \in \Delta$ together with (ii) implies $(\hat{x}-x)$ $\cdot A(x-\hat{x})>0$ for all $x$ and hence

$$
\begin{aligned}
\xi \cdot A \xi<0 \quad \forall \xi \in \mathrm{R}_{0}^{n} & =\left\{\xi \in \mathrm{R}^{n}: \sum_{i} \xi_{i}=0\right\} \\
\text { with } \xi & \neq 0 .
\end{aligned}
$$

The condition (16) says that the mean payoff $x \cdot A x$ is a strictly concave function on $\Delta$. Conversely, games satisfying (16) have a unique ESS (possibly on the boundary) which is also the unique Nash equilibrium of the game, see Hofbauer and Sigmund (1998, p. 72). The slightly weaker condition

$$
\xi \cdot A \xi \leq 0 \quad \forall \xi \in \mathrm{R}_{0}^{n}
$$

includes also the limit case of zero-sum games and games with an interior equilibrium that is a neutrally stable strategy (i.e., equality is allowed in (ii)) as defined by Maynard Smith (1982), see also Weibull (1995). Games satisfying (17) need no longer have a unique equilibrium, but the set of equilibria is still a nonempty convex subset of $\Delta$.

\subsection{The best reply dynamics}

Theorem 4.1 Consider a game with (17). Then the convex set of its equilibria is globally asymptotically stable for the best-response dynamics (13). In particular, if $\hat{x}$ is an interior ESS then $\hat{x}$ is globally asymptotically stable for (13). Moreover all (piecewise linear) paths reach $\hat{x}$ in finite time.

Proof. Consider the convex function

$$
V(x)=\max _{i}(A x)_{i}-x \cdot A x
$$

which satisfies $V(x) \geq 0$, and $V(x)=0$ if and only if $x$ is a Nash equilibrium. Hence the set of equilibria is convex. Along a linear piece $\dot{x}=b-x$ of a solution of (13), one has $V=(b-x) \cdot A x$ and 


$$
\begin{aligned}
\dot{V} & =-\dot{x} \cdot A x+(b-x) \cdot A \dot{x}= \\
& =-(b-x) \cdot A x+(b-x) \cdot A(b-x) .
\end{aligned}
$$

Since the second term is nonpositive due to (17) we get $\dot{V} \leq-V \leq 0$. This shows the global asymptotic stability of the equilibrium set $\{\mathrm{V}=0\}$. If $\hat{x}$ is an interior ESS then the second term in (19) is negative and even bounded away from 0 since $b$ is on the boundary of $\Delta$ unless $x=\hat{x}$. Hence $V(x(t))$ decreases strictly and reaches the value 0 in finite time.

Actually more can be shown, see Hofbauer (1995): If (16) holds, then there is a unique solution to (13) through each initial value. If $\hat{x}$ is an ESS on the boundary of $\Delta$ then it is locally asymptotically stable for (13). The ESS assumption can be considerably relaxed. Moreover, these local and global stability assertions hold also for Brown's discrete fictitious play process.

As a special case we recover a version of Brown's (1951) result: For a symmetric zero-sum game, all solutions of (13) converge to the set of equilibria.

\subsection{The smoothed BR dynamics}

We start with a simple lemma which shows that the directional derivative of the modified payoff function $F(z, x)$ at $z=x$ pointing towards $z=b_{\varepsilon}(x)$ is positive at every $x$ (with the obvious exception of an $\varepsilon$-equilibrium where $\left.b_{\varepsilon}(x)=x\right)$. $\left(\partial_{1}\right.$ denotes the derivative with respect to the first variable.)

\section{Lemma}

$$
\partial_{1} F(x, x)\left(b_{\varepsilon}(x)-x\right) \geq 0,
$$

with equality only when $b_{\varepsilon}(x)=x$.

Proof. Since $\partial_{1} F\left(b_{\varepsilon}(x), x\right)=0$ from (9), the claim is equivalent to

$$
\left(\partial_{1} F\left(b_{\varepsilon}(x), x\right)-\partial_{1} F(x, x)\right)\left(b_{\varepsilon}(x)-x\right) \leq 0 .
$$

This follows easily from the fact that $F(\cdot, x)$ is a strictly concave function on int $\Delta$ in its first variable.

Theorem 4.2 Let the game satisfy (17). Then there is a unique $\varepsilon$-equilibrium $\hat{x}_{\varepsilon}$ which is globally asymptotically stable for the smoothed BR dynamics
(14). In particular, an interior ESS $\hat{x}$ generates globally asymptotically stable equilibria $\hat{x}_{\varepsilon}$ of (14) that approach $\hat{x}$ as $\varepsilon \rightarrow 0$.

Proof. Consider the function

$$
V(x)=F\left(b_{\varepsilon}(x), x\right)-F(x, x)=\max _{z \in \Delta} F(z, x)-F(x, x)
$$

which is nonnegative by definiton of $b_{\varepsilon}$ and vanishes precisely at points $x \in \Delta$ with $b_{\varepsilon}(x)=x$, i.e. the rest points of (14). Moreover, $V$ is strictly convex and hence attains its minimum value 0 at a unique such point. Then

$$
\begin{aligned}
V= & \partial_{1} F\left(b_{\varepsilon}, x\right) \beta_{\varepsilon}-\partial_{1} F(x, x) \dot{x}+\partial_{2} F\left(b_{\varepsilon}, x\right) \dot{x}- \\
& -\partial_{2} F(x, x) \dot{x} .
\end{aligned}
$$

The first expression vanishes, since $\partial_{1} F\left(b_{\varepsilon}(x), x\right)$ $=0$ by definition (9) of $b_{\varepsilon}(x)$, the second term is taken care of by the Lemma, and the last two terms simplify to $\left(b_{\varepsilon}-x\right) \cdot A \dot{x}=\dot{x} \cdot A \dot{x}$ which is nonpositive thanks to the negative semidefiniteness assumption (17). Hence $\dot{V} \leq 0$ with equality only at the unique $\varepsilon$-equilibrium, and $V$ is a global Lyapunov function. The convergence of $\hat{x}_{\varepsilon} \rightarrow \hat{x}$ as $\varepsilon \rightarrow 0$ follows from the closed graph of the BR-correspondence, see section 2.2.

The local stability of interior ESS was already shown by Hopkins (1999). From his elegant expression for the linearization of $(14), \dot{\xi}=\frac{1}{\varepsilon} C(\hat{x}) A \xi-\xi$, with $C$ a symmetric positive definite matrix depending on $v$, one can easily derive the following converse result: If an (isolated) interior equilibrium $\hat{x}$ generates nearby (locally asymptotically) stable $\varepsilon$-equilibria $\hat{x}_{\varepsilon}$ after small perturbation with all admissable choices of $v$ then $A$ satisfies the negative semidefiniteness condition (17). Hence $\hat{x}$ is a neutrally stable strategy, i.e. 'almost' an ESS.

\subsection{The Brown-von Neumann-Nash dynamics}

Theorem 4.3 For every game with (17) the set of its equilibria is globally asymptotically stable for (15). In particular, an interior ESS is globally asymptotically stable for (15).

Proof. Let us first compute the derivative of $k_{i}$. If $k_{i}(x)>0$ then 


$$
\begin{aligned}
k_{i}= & (A \dot{x})_{i}-x \cdot A \dot{x}-\dot{x} \cdot A x \\
& =\bar{k}\left[(A(q-x))_{i}-x \cdot A(q-x)-(q-x) \cdot A x\right]
\end{aligned}
$$

with $\bar{k}=\sum_{i} k_{i}$ and $q_{i}=k_{i} / \bar{k}$. Consider now the convex function $V=\frac{1}{2} \sum_{i} k_{i}(x)^{2}$. Then

$$
\begin{aligned}
\dot{V} & =\sum_{i} k_{i} k_{i}=\bar{k} \sum_{i} q_{i} \dot{k}_{i} \\
& =\bar{k}^{2}[q \cdot A(q-x)-x \cdot A(q-x)-(q-x) \cdot A x] \\
& =\bar{k}^{2}(q-x) \cdot A(q-x)-\bar{k} \dot{x} \cdot A x .
\end{aligned}
$$

The first term is nonpositive due to (17), and the second term is nonnegative because of

$$
\begin{aligned}
\dot{x} \cdot A x & =\sum_{i} k_{i}(A x)_{i}-\bar{k} x \cdot A x= \\
& =\sum_{i} k_{i}\left[(A x)_{i}-x \cdot A x\right]=\sum_{i} k_{i}^{2} \geq 0 .
\end{aligned}
$$

Hence $\dot{V}(x)<0$ for all $x$ outside the equilibrium set, and $V$ is a global Lyapunov function.

This theorem contains also the classical result of Brown and von Neumann (1950) as special case: For a zero-sum game $A=-A^{T}$, the set of equilibria is globally asymptotically stable for (15).

For ESS on the boundary, local stability can be shown at least under an additional regularity assumption. I refer to Berger and Hofbauer (1998) for this and many other results on the Brown-von Neumann-Nash dynamics.

\section{Fisher's fundamental theorem}

We consider now games with a symmetric payoff matrix $A=A^{T}\left(a_{i j}=a_{j i}\right.$ for all $\left.i, j\right)$. Such games are known as partnership games and potential games. The basic population genetic model of Fisher is equivalent to the replicator dynamics for such games, which is then a gradient system with respect to the Shahshahani metric and the mean payoff as potential, see e.g. Hofbauer and Sigmund (1998). The resulting increase of mean fitness or mean payoff $x \cdot A x$ in time is often referred to as the fundamental theorem of natural selection. This statement about the replicator dynamics generalizes to the dynamics considered in this paper.
The generalization is based on the concept, defined by Swinkels (1993), of a (myopic) adjustment dynamics which satisfies $\dot{x} \cdot A x \geq 0$ for all $x \in \Delta$, with equality only at equilibria. If $A=A^{T}$ then the mean payoff $x \cdot A x$ is increasing for every adjustment dynamics since $(x \cdot A x)^{\circ}=2 \dot{x} \cdot A x \geq 0$. It is obvious that the best-response dynamics (13) is an adjustment dynamics and, as shown in (21), (15) is as well (see (27) below for its generalization to (23)). As a consequence, we obtain the following result.

Theorem 5.1 For every potential game $A=A^{T}$, every trajectory of every adjustment dynamics (in particular (13), (15) and (23)) converges to (a connected set of) equilibria.

For the perturbed BR dynamics there is the following modification, thanks to the lemma in section 4.2 .

Theorem 5.2 For every potential game, the function $P(x)=1 / 2 x \cdot A x+\varepsilon v(x)$ increases monotonically along solutions of (14) and hence every solution converges to (a connected set) of E-equilibria.

Proof.

$$
\dot{P}=\dot{x} \cdot A x+\varepsilon v^{\prime}(x) \dot{x}=\partial_{1} F(x, x) \dot{x} \geq 0
$$

according to the Lemma, with equality only at $\varepsilon$-equilibria.

\section{Generalized "Brownian motion"}

Let $f: \mathrm{R}_{+} \rightarrow \mathrm{R}_{+}$be a continuous function with

$$
f(0)=0 \text { and } f(u)>0 \text { for } u>0 .
$$

Then, recalling the definiton (12) of $k_{i}(x)$, we consider the dynamics

$$
\dot{x}_{i}=f\left(k_{i}\right)-x_{i} \sum_{j=1}^{n} f\left(k_{j}\right) .
$$

Theorem 6.1 For a game with (17), the set of equilibria is globally asymptotically stable for (23). In particular, an interior ESS is globally asymptotically stable for (23). 
Proof. Consider the function

$$
V(x)=\sum_{i} F\left(k_{i}(x)\right)
$$

where $F$ denotes an antiderivative of $f$, i.e. $F^{\prime}=f$. Using the abbreviations $\bar{f}=\sum_{i} f\left(k_{i}\right)$ and $\widetilde{f}_{i}=f\left(k_{i}\right) / \bar{f}$, we have (whenever $k_{i}(x)>0$ )

$$
k_{i}=\bar{f}\left[\left(e_{i}-x\right) \cdot A(\tilde{f}-x)-(\tilde{f}-x) \cdot A x\right]
$$

and

$$
\begin{aligned}
\dot{V} & =\sum_{i} F^{\prime}\left(k_{i}\right) \dot{k}_{i}=\sum_{i} f\left(k_{i}\right) \dot{k}_{i}=\bar{f} \sum_{i} \tilde{f} k_{i} \\
& =\bar{f}^{2}[(\tilde{f}-x) \cdot A(\tilde{f}-x)-(\tilde{f}-x) \cdot A x]= \\
& =\dot{x} \cdot A \dot{x}-\bar{f} \dot{x} \cdot A x
\end{aligned}
$$

which is negative as in the proof of Theorem 4.3. For the second term observe

$$
\begin{aligned}
\dot{x} \cdot A x & =\sum_{i} f\left(k_{i}\right)(A x)_{i}-\bar{f} x \cdot A x= \\
& =\sum_{i} f\left(k_{i}\right)\left[(A x)_{i}-x \cdot A x\right]= \\
& =\sum_{i} f\left(k_{i}\right) k_{i} \geq 0
\end{aligned}
$$

because of (22). Equality holds iff $k_{i}=0$ for all $i$, i.e., if $x$ is a Nash equilibrium.

An important special case of (22) is $f(u)=u^{\alpha}$ where $\alpha>0$, see Weibull (1994). For $\alpha=1$, i.e. $f(u)$ $=u$ we recover the BNN dynamics (15) and in the limit $\alpha \rightarrow \infty$ (23) tends (after normalization) to the BR dynamics. Hence (23) is a joint generalization of the two dynamics introduced by G. W. Brown. We note that the Lyapunov function (24) reduces to $V=\frac{1}{\alpha+1} \sum_{i} k_{i}^{\alpha+1}$ for $f(u)=u^{\alpha}$ and converges in the limit $\alpha \rightarrow \infty$ (after normalization) to $V(x)=\max _{i}$ $k_{i}(x)=\max _{i}(A x)_{i}-x \cdot A x$. So it recovers the two Lyapunov functions used for the proofs of Theorem 4.1 and 4.3.

\section{Conclusion}

Two families of game dynamics have been presented for which interior ESS are globally asymptotically stable: the family (23) which includes the Brown-von Neumann-Nash dynamics as the sim- plest special case, and the perturbed BR dynamics (14). Both contain the BR dynamics (Brown's fictitious play) as limit case. Both families are large, infinite dimensional in fact, as an arbitrary function $(f$ in the first case, $v$ in the second case) can be chosen. The replicator dynamics (together with a few of its relatives) is the only other dynamics known so far to enjoy this fundamental property that an interior ESS is globally asymptotically stable. The global stability results presented here are even stronger than those for the replicator dynamics, as they hold under the weaker assumption of (17). In particular they include the limit case of zero-sum games for which the replicator dynamics yields only neutral stability for interior equilibria.

Apparently Maynard Smith's ESS concept has to offer as much to these rationalistic game dynamics originating in Brown's and Nash's work as to the evolutionary dynamics of replicators.

\section{References}

Berger, U. and Hofbauer, J. (1998): The Nash Dynamics. Preprint.

BROWN, G. W. (1949): Some notes on computation of games solutions. RAND Report P-78.

BROWN, G. W. (1951): Iterative solution of games by fictitious play. In Activity Analysis of Production and Allocation. Wiley, New York, pp. 374-376.

Brown, G. W. and VON NEUMANN, J. (1950): Solutions of games by differential equations. Ann. Math. Studies 24: 73-79.

CRESSMAN, R. (1997): Local stability of smooth selection dynamics for normal form games. Math. Social Sciences 34:1-19.

FUDENBERG, D. and LEVINE, D. K. (1998): The Theory of Learning in Games. MIT Press.

GILBOA, I. and MATSUI, A. (1991): Social stability and equilibrium. Econometrica 59:859-867.

HofBAUER, J. (1995): Stability for the Best Response Dynamics. Preprint.

HofBAUER, J. and SigMUND, K. (1998): Evolutionary Games and Population Dynamics. Cambridge University Press.

HoPKINS, E. (1999): A note on best response dynamics. Games Econ. Behav. 29:138-150.

KAKUTANI, S. (1941): A generalization of Brouwer's fixed point theorem. Duke J. Math. 8:457-459.

LEONARD, R. J. (1994): Reading Cournot, reading Nash: The creation and stabilisation of the Nash equilibrium. The Economic Journal 104:492-511.

MAYNARD SMITH, J. (1982): Evolution and the Theory of Games. Cambridge University Press.

MAYNARD SMITH, J. and PRICE, G. (1973): The logic of animal conflicts. Nature 246:15-18. 
NASH, J. (1950a): Equilibrium points in $\mathrm{N}$-person games. Proc. Natl. Ac. Sci. 36:48-49.

NASH, J. (1950b): Non-cooperative Games. Dissertation, Princeton University, Dept. Mathematics.

NASH, J. (1951): Non-cooperative games. Ann. Math. 54: 287-295.

[NASH, J.] The Work of John Nash in Game Theory. Nobel Seminar Dec 8, 1994. J. Econ. Theory 69 (1996):153-185.
Owen, G. (1982): Game Theory. 2nd ed. Academic Press, Orlando.

SwINKELS, J. M. (1993): Adjustment dynamics and rational play in games. Games Econom. Behav. 5:455-484.

WeIBULL, J. W. (1994): The Mass-action Interpretation of Nash Equilibrium. Preprint, Stockholm. Revised 1995. Partly published in J. Econ. Theory 69 (1996).

WeIBULl, J. W. (1995): Evolutionary Game Theory. MIT Press. 